\title{
RADIATIVE SHOCKS IN ASTROPHYSICS AND THE LABORATORY
}

\author{
R. PAUL DRAKE \\ Atmospheric Oceanic and Space Sciences, University of Michigan, Ann Arbor, MI, USA; \\ E-mail: rpdrake@umich.edu
}

(Received 28 April 2004; accepted 25 May 2004)

\begin{abstract}
This paper explores the variations in radiative shock behavior originating from the properties of the system containing the shock. Specifically, the optical depth of the upstream region and the downstream region both affect the behavior of radiative shocks. Optically thick systems such as stellar interiors or supernovae permit only limited shock-induced increases in density. At the other limit, the radiation and shock dynamics in optically thin systems permits the post-shock density to reach arbitrarily large values. The theory of the shock structure is summarized for systems in which the upstream region is optically thin, common to some astrophysical systems and a number of experiments.
\end{abstract}

Keywords: shock waves, radiative shock waves, laboratory astrophysics

\section{Introduction}

A radiative shock is one in which the structure of the density and temperature is affected by radiation from the shock-heated matter. Here we explore the properties of such shocks in a large context - that of all (nonrelativistic) laboratory experiments and astrophysical systems. In order for a shock to be radiative in any medium, it must at minimum be fast enough that the radiative fluxes, which scale as the fourth power of the temperature, exceed the material energy fluxes, which scale as the three-halves power of temperature. In the nonradiative regime, the immediate postshock temperature $T_{\mathrm{i}}$ is given by

$$
k_{\mathrm{B}} T_{\mathrm{i}}=\frac{2(\gamma-1)}{(\gamma+1)^{2}} \frac{A m_{\mathrm{p}}}{(Z+1)} u_{\mathrm{s}}^{2},
$$

in which $k_{\mathrm{B}}$ is the Boltzmann constant, $\gamma$ is the usual ratio of specific heats, $A$ is the average atomic weight, $m_{\mathrm{p}}$ is the proton mass, and $u_{\mathrm{s}}$ is the shock velocity. The average number of electrons that share energy with each ion is $Z$, which can be a source of difficulty in two senses.

First, the shock heats the ions and then the electrons and ions equilibrate, so that in sufficiently low-density matter $Z$ might be zero immediately following the density jump. Thus, in general, one may need to allow separate temperatures for ions and electrons. It is the electrons, though, that couple significantly to the radiation. Here 
for simplicity we assume immediate equilibration of ions and electrons. In practice, this means that the region just behind the shock (the jump in density and ion temperature) where ions and electrons equilibrate is ignored. The radiation from this equilibration zone increases as the fourth power of the electron temperature, so that most of the equilibration zone is not a significant contributor to the radiation dynamics.

Second, $Z$ may be temperature-dependent if the medium is not fully ionized. This is particularly true for Xenon, a common material in laboratory radiative shock experiments. Here we use for Xenon the approximation $Z=20 \sqrt{T_{\mathrm{i}}}$, with the initial post-shock electron (and ion) temperature $T_{\mathrm{i}}$ in $\mathrm{keV}$. This can be derived from the Saha equation and thus assumes equilibrium (Drake, 2005). It converts Eq. (1) to a cubic equation relating temperature and shock velocity, easily dealt with in today's era of computational algebra.

One can then ask when radiative fluxes or pressure matter in shock-heated systems. This ultimately can be a complicated problem, as Eq. (1) breaks down when radiation pressures begin to become significant, and as the importance of radiative effects depends on the structure and opacity of the system. To obtain a preliminary assessment, we assume that Eq. (1) applies throughout, and consider optically thick conditions so that radiation temperatures equal material temperatures. This second assumption is valid for some astrophysical systems and some laboratory experiments, but not others. We explore this further in later sections. Under these assumptions, one can ask when the radiative flux, $F_{\mathrm{R}}=\sigma T_{\mathrm{i}}^{4}$, equals the material flux, $F_{\mathrm{m}}=\rho_{o} c_{\mathrm{v}} T_{\mathrm{i}} u_{\mathrm{s}}$, in which the upstream density is $\rho_{o}$, the heat capacity at constant volume is $c_{\mathrm{v}}$, and the Stefan-Boltzmann constant is $\sigma$. One can also ask when the radiation pressure, $P_{\mathrm{R}}=4 \sigma T_{\mathrm{i}}^{4} /(3 c)$ equals the material pressure, $p$.

One finds the results shown in Figure 1. Curves are shown for xenon and for $\mathrm{CH}$ as labeled. In the space to the right of each curve, the radiation parameter $\left(F_{\mathrm{R}}\right.$ or $\left.p_{\mathrm{R}}\right)$ exceeds the corresponding material parameter for the given material. One sees that there is roughly an order of magnitude in shock velocity over which radiation fluxes dominate the energy transport but radiation pressures are unimportant. As radiative conditions have been difficult to reach in experiments, this regime has been the most common one to date. In general, one sees that in optically thick systems shock velocities of tens to hundreds of $\mathrm{km} / \mathrm{s}$ are required to reach radiative regimes.

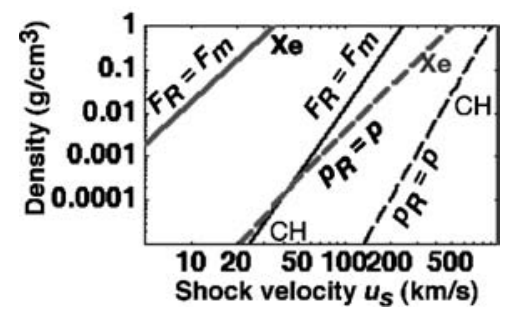

Figure 1. Regimes of radiative effects for optically thick media. The lines show boundaries, with stronger radiative effects to the right. 
The shock velocities required to reach radiative regimes are larger in optically thin systems. The radiative flux from an optically thin system, for thermal emission, equals $\varepsilon F_{\mathrm{R}}$, where $\varepsilon$ is the optical depth of the system. However, many optically thin systems, especially in astrophysics, produce primarily line emission, in which case $\varepsilon$ would be an appropriate average over the spectral variation of the optical depth and thermal spectrum. Indeed, astrophysicists more often work with a "cooling function" to characterize the power radiated, and in optically thin astrophysical systems the optical depth decreases so rapidly with increasing temperature that the radiation tends to decrease as electron temperature $T_{\mathrm{e}}$ increases up to $T_{\mathrm{e}}$ of order 1 $\mathrm{keV}$. Curiously, the shock velocity required for radiation fluxes to be significant is increased by finite optical depth into the range of $>100 \mathrm{~km} / \mathrm{s}$, which is just where it is for laboratory experiments with foams or dense gas. Similarly, the radiation pressure in an optically thin system is $\varepsilon p_{R}$, This makes the radiation-dominated regime genuinely difficult to reach in experiments. From Figure 1, one might seek to reach this regime at lower shock velocity by using low-density xenon gas. However, the size of the experiment must increase as some (regime-dependent) power of the decrease in density to hold the optical depth fixed. Claims that specific regimes have been reached in experiments with gas must be carefully justified.

\section{Radiative Precursors and the Definition of the Shock Transition}

We showed in Section 1 that a steady shock must reach some velocity if radiative energy fluxes are to exceed material energy fluxes, with the exact value dependent on conditions. By the time this occurs, the radiation is affecting the medium upstream of the region across which the rapid density increase takes place. There are two ways to think about this development. On the one hand, if one views the medium as infinite (measured in optical depths), then one may take the point of view that the radiation alters the structure of the shock transition, extending it in space over a (potentially large) number of radiation mean free paths. In this case one will speak of the "shock" as the entire region between a distant, undisturbed upstream region and a distant, steady-state downstream region.

On the other hand, it may be that the optical depth from the shock to "infinity" is small, as is the case in certain classes of astrophysical shocks. What is meant here is that the sum of radiation from distant sources and radiation returning to the shock from any upstream, shock-heated matter is negligible. Beyond this, the downstream region might not be optically thick, as is the case for example in the shocks driven by supernova remnants and in some short-lived experiments. Whenever the entire region affected by radiation from the shock is not well isolated from other influences, it seems more natural to speak of the "shock" as the region across which the rapid density increase takes place, often referred to as the viscous shock transition or viscous density jump. This use of "shock" is more common in discussions of optically thin astrophysical shocks. In this case, the interactions 
of the radiation and the surrounding medium affect both the upstream and the downstream conditions.

In either case, it is useful to discuss the development of radiative effects ahead of the shock, or of a radiative precursor, with three levels of sophistication. On the first level, one can say that a radiative precursor will be present when the flux of ionizing photons radiated ahead of the shock equals the flux of ionized neutral atoms incident on the shock (Keiter et al., 2002). This point of view is that one will certainly see heating of the upstream medium when all (or most) of the incoming atoms are ionized. This balance can be expressed by

$$
2.3 \times 10^{23} \varepsilon_{\mathrm{u}} \varepsilon_{\mathrm{d}} \eta T_{\mathrm{i}}^{3}>\frac{\rho_{o} u_{\mathrm{s}}}{\left(A m_{\mathrm{p}}\right)},
$$

where $T_{\mathrm{i}}$ is in $\mathrm{eV}, \rho_{o}$ is the mass density in $\mathrm{g} / \mathrm{cm}^{3}$, and $\eta$ is the fraction of the photons that are ionizing. The emissivity (or the optical depth for small optical depth) of the downstream region is $\varepsilon_{\mathrm{d}}$ and the emissivity (equal to the absorptivity) of the upstream region is $\varepsilon_{\mathrm{u}}$. The downstream emissivity determines what fraction of blackbody emission is actually produced and the upstream emissivity determines what fraction of these photons is absorbed. The corresponding threshold for a radiative precursor, using Eq. (1) for $T_{\mathrm{i}}$, is $u_{\mathrm{s}}>270\left[\rho_{o} /\left(\varepsilon_{\mathrm{d}} \varepsilon_{\mathrm{u}} \eta\right)\right]^{1 / 5} \mathrm{~km} / \mathrm{s}$. For shock velocities above $50 \mathrm{~km} / \mathrm{s}$, relevant to many laboratory experiments, nearly all the photons are ionizing. In laboratory experiments with dense gases or foams, both emissivities may be of order unity as well. For low-density astrophysical systems, with $\rho_{o}$ of order $10^{-24} \mathrm{~g} / \mathrm{cm}^{3}$, obtaining a radiative precursor will require first of all that the post-shock temperature be high enough to obtain a sufficient fraction of ionizing photons and beyond that on the optical depth of the system.

On a second level, one can treat the precursor as a nonlinear radiation diffusion wave. Since such waves have a length-dependent velocity, one can argue that, in steady state, the precursor length ahead of a shock must be such that the diffusion wave velocity equals the shock velocity.(Mihalas and Weibel-Mihalas, 1999). A diffusion wave from a constant-temperature source has a length $L$ that scales with time $t$ as $L=K \sqrt{t}$, in which the coefficient $K$ depends on the scaling of the material opacity with density and temperature, and thus does depend on the shock velocity. From the velocity matching argument just given one finds $L=K^{2} /\left(2 u_{\mathrm{s}}\right)$. One finds for example that in experiments with xenon the precursor becomes very large as the velocity increases above $10 \mathrm{~km} / \mathrm{s}$. However, the timescale required for the precursor to reach its steady state, found from the above to be $t=K^{2} /\left(4 u_{\mathrm{s}}^{2}\right)$, also increases as the precursor length increases. The consequence is that real experiments, and even some astrophysical systems, may not establish a steady state precursor during their lifetime.

On a third level, one can consider finer details. An important issue in some contexts will be that many such shocks, in astrophysics and in experiments with 
low-density gases, are not planar. Spherical and even cylindrical models would be useful tools for these cases. For a specific experiment, one might consider the actual time dependence of the shock velocity and the consequent radiation source, as Fleury et al. have done (Fleury et al., 2002). Details beyond those treated by diffusion models can also be considered. Zel'dovich and Razier (1966), for example, argue that the leading edge of such a diffusion wave cannot be in equilibrium, with the result that there is a low-radiation-temperature foot ahead of the diffusion wave. However, this foot is decoupled from the material, and so is not a precursor in the sense that the state of the upstream medium is affected.

\section{Regimes of Radiative Shocks}

The category of radiative shocks includes a wide range of behavior. For example, in some regimes the density increase across the viscous transition and subsequent radiative cooling layer (if any) can be limited to values less than 10, while in other regimes it can be formally unbounded so long as the plasma is not radiation-dominated. (The $\gamma$ of radiation is $4 / 3$, so the density jump in any radiation-dominated plasma is 7.) One way to identify types of radiative shocks, and to classify their behavior, is to plot them in a space defined by the optical depth of the upstream and downstream regions. Figure 2 shows a qualitative depiction of this space. We next briefly discuss each of the four labeled regions.

\subsection{THICK-THICK SHOCKS}

In regime $\mathrm{A}$, both the downstream and the upstream region are optically thick. This is the realm in which it makes the most sense to treat the viscous density increase and all the radiative effects as part of a single, extended, shock structure, and in which many of the features of this structure can be found from a theory that assumes the medium to be in LTE everywhere. This is also the realm that is treated at length

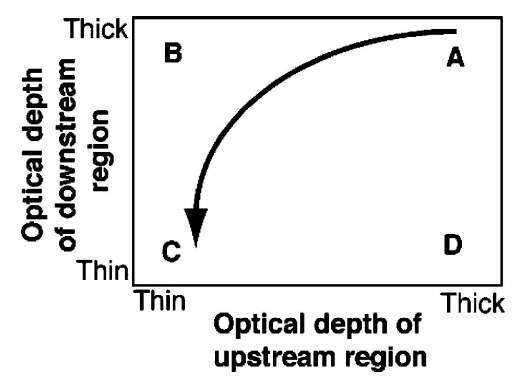

Figure 2. The types of radiative shocks can be identified in a space based on optical depth. The four regimes corresponding to the corners of this plot are discussed in the text. The curve shows the qualitative trajectory of a supernova blast wave. 
in books that discuss radiation hydrodynamics, such as those by Zel'dovich and Razier (1966) and Mihalas and Weibel-Mihalas (1999) and discussed as well in some recent theory papers (Boireau et al., 2004; Bouquet et al., 2000). There is a definite limit on the density ratio of such shocks. For typical ideal gases with $\gamma \sim 5 / 3$, the density ratio never exceeds 7 . (In some complex atoms at low temperatures, the ratio can be somewhat larger (Boireau et al., 2004).) In addition, in some regimes the density transition is continuous, with no localized jump. Astrophysical environments in which such shocks exist are necessarily both hot and dense. Shocks in stellar interiors are of this type, as are the blast waves within the exploding star in supernovae. Such shocks may also exist within some compact objects, but their treatment would have to be relativistic. It is difficult, however, to imagine laboratory experiments in this regime other than transiently and in special cases. One difficulty is that the precursor length increases so strongly with shock velocity that one could not produce a measurable precursor of finite length over any variation of experimental parameters. One ought to obtain a slower scaling of length with velocity in spherical geometry, but at the low densities where such experiments are straightforward the downstream region is far from being optically thick. (Note that a finite precursor is not necessarily optically thick in the sense that matters here; an optically thick precursor would have many optical depths between the density jump and the heat front.)

\subsection{THICK-THIN SHOCKS}

In regime B the downstream region is optically thick but the upstream region is thin. We discuss the theory of this regime in the next section. There is a cooling layer downstream of the viscous shock transition, followed by a steady downstream final state. This regime is common in experiments (Bouquet et al., 2004; Bozier et al., 1986; Fleury et al., 2002; Keiter et al., 2002; Koenig et al., 2001; Reighard et al., 2004), in which an optically thick piston (and in some cases optically thick shocked material) drives a radiative shock into a medium whose depth is small compared to the steady-state precursor length. The upstream medium is then quickly heated so that it becomes optically thin. Astrophysical examples of such systems include the blast wave in a supernova as it emerges from the star (Ensman and Burrows, 1992) and the accretion shocks produced during star formation (Calvet and Gullbring, 1998; Hujeirat and Papaloizou, 1998; Lamzin, 1999).

\subsection{THIN-THIN SHOCKS}

In regime $\mathrm{C}$ both downstream and upstream regions are optically thin. Such shocks are the most commonly observed in astrophysics, in part because they are easy to see (as the radiation escapes). Supernova remnant (SNR) shocks in dense enough environments are of this type - it is thought that Type II supernovae from red 
supergiants produce such conditions (Chevalier, 1997). Many shock-cloud interactions including some of those driven by SNR shocks are also of this type. Shocks that propagate up jets (or are driven by clumps propagating up jets) may be of this type (Hartigan, 2003). In such shocks, the entire downstream region is a radiative cooling layer, and it ends (in large enough systems) when the downstream temperature reaches a value determined by local sources and losses of energy rather than by the shock. The density increase associated with such shocks is formally unbounded in the sense that it is limited only by external factors, such as the compression of an initially negligible magnetic field or the presence of a limiting temperature due to other energy sources. These shocks have much in common with the radiative phase of old supernova remnants (Blondin et al., 1998), which occurs when an SNR shock cools enough that the slope of the radiative flux (astrophysicists would say the cooling function) with temperature changes from positive to negative, enabling the entire shocked region to rapidly radiate away its energy and the density to increase by orders of magnitude. Some experiments, with shocks in sufficiently low-density gases, may produce these conditions (see, for example (Grun et al., 1991)), but this question deserves more detailed examination than we can provide here. We discuss the theory of these shocks briefly in the next section. It is also discussed by Shu (1992).

\subsection{THIN-THICK SHOCKS}

Regime D is difficult to access, and perhaps is only seen in a transient sense. If the upstream region is optically thick, then the downstream, shocked material is likely to become optically thick as it accumulates and radiatively cools. Ignoring the increase in optical depth, such a system could produce a very dense shocked layer as it continued to lose energy in the downstream direction. Two transient examples are certain shock-cloud collisions and certain experiments. A shock-cloud collision in which the cloud was dense enough and large enough to be optically thick for some time would be of this type. The collision of SNR 1987A with its inner "ring" may be of this type (Borkowski et al., 1997), especially if the ring turns out to be a disk. An experiment might be in this regime while a hot, thin layer of gas drives a shock through a much larger volume of gas, as seems to be the case in some experiments done with xenon gas at low densities. (See for example the papers by F. Hansen and by T. Ditmire in this issue.) All these cases seem likely to transition to the thin-thin regime if driven harder or longer, and they may never develop a thick upstream region in the sense discussed above.

\section{Structure of the Radiative Cooling Layer}

In shocks with an optically thin upstream region, the structure of the radiative cooling layer behind the shock is of interest. One would like to understand its size, 

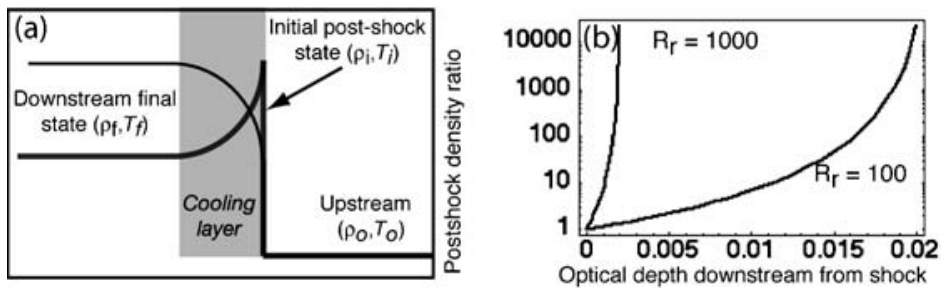

Figure 3. (a) Structure of the radiative cooling layer. (b) Density profiles for a thin-thin shock.

its shape, and the maximum density that is reached. For this purpose, one can work with a fluid theory including radiation transport. However, for the radiation one cannot assume LTE because the material temperature turns out to change on scales much smaller than a radiation mean-free-path.

Under these assumptions, the structure of the cooling layer is shown in Figure 3(a). There is some upstream density, pressure and temperature. The upstream region has perhaps been heated by the precursor but is not evolving further in the optically thin limit, and contributes a negligible radiation heat flux. At the viscous shock, there is a density jump and an increase in temperature, with the immediate post-shock (and post-electron-ion-equilibration) temperature given by Eq. (1). Downstream from this is the cooling layer, whose behavior we wish to calculate. The cooling layer ends when the temperature and density reach their final values, $T_{\mathrm{f}}$ and $\rho_{\mathrm{f}}$, respectively. If the final temperature is nonzero, then the downstream radiation from the cooling layer must balance the upstream radiation from the steady downstream layer, which is $\sigma T_{\mathrm{f}}^{4}$. This upstream radiation flux must also equal half the loss of downstream material energy flux between the shock and the final downstream state, as this is the only source of energy for the radiation from the cooling layer (which radiates both upstream and downstream). This flux balance condition can be used to determine the final post-shock density and temperature.

The equations that describe the evolution of the cooling layer are as follows. The fluid energy equation, in steady state, is

$$
\nabla \cdot\left[\rho \mathbf{u}\left(\varepsilon+\frac{u^{2}}{2}\right)+p \mathbf{u}\right]=-\nabla \cdot \mathbf{F}_{\mathrm{R}}
$$

in which the velocity is $\mathbf{u}$, the density is $\rho$, the pressure is $p$, and the internal energy $\varepsilon$ will be taken to equal $p /(\gamma-1)$. The radiation flux is $\mathbf{F}_{\mathrm{R}}$. Using the momentum and continuity equations, one can express the equation of state for some location, relative to the immediate post-shock density $\rho_{\mathrm{i}}$ and temperature $T_{\mathrm{i}}$, as

$$
\frac{T}{T_{\mathrm{i}}}=\frac{\gamma+1}{2} \frac{\rho_{\mathrm{i}}}{\rho}\left[1-\frac{\gamma-1}{\gamma+1} \frac{\rho_{\mathrm{i}}}{\rho}\right]
$$


We express the radiation loss in the "transport" approximation corresponding to isotropic scattering, taking $-\nabla \cdot \mathbf{F}_{\mathrm{R}}=4 \pi \kappa\left(B-J_{\mathrm{R}}\right)$, in which $\kappa$ is a frequencyaveraged absorption coefficient, approximately equal to the Planck mean opacity, $B$ is the intensity of radiation within a black body at the local electron temperature, and $J_{\mathrm{R}}$ is the angularly averaged radiation intensity. From these relations one can obtain

$$
\left[1-\frac{(\gamma-1)}{\gamma} \frac{\rho_{\mathrm{i}}}{\rho}\right] \frac{\partial}{\partial \tau}\left(\frac{\rho_{\mathrm{i}}}{\rho}\right)=-R_{r}\left(\left(\frac{T}{T_{\mathrm{i}}}\right)^{4-n}-\left(\frac{\pi J_{\mathrm{R}}}{\sigma T_{\mathrm{i}}^{4}}\right)\left(\frac{T}{T_{\mathrm{i}}}\right)^{-n}\right)\left(\frac{\rho_{i}}{\rho}\right)^{m}
$$

in which the optical depth variable $\tau=z \kappa_{\mathrm{i}}$, with $\kappa_{\mathrm{i}}$ being the value of the opacity at $T_{\mathrm{i}}$ and $\rho_{\mathrm{i}}$. The opacity is taken to vary as powers of $1 / T$ and $\rho$, with exponents $n$ and $m$, respectively. The parameter that characterizes the importance of the radiation is $R_{r}$, given by

$$
R_{r}=\frac{4(\gamma+1)}{\gamma} \frac{\sigma T_{\mathrm{i}}^{4}}{\rho_{o} u_{\mathrm{s}}^{3}}
$$

and approximately equal to the ratio of upstream energy flux produced by a blackbody at temperature $T_{\mathrm{i}}$ to the energy flux of heated material downstream from the shock. When this exceeds roughly unity, there evidently must be significant cooling. The quantity $J_{\mathrm{R}}$ in Eq. (5) is the radiation intensity within the final downstream layer, and equals $\sigma T_{\mathrm{f}}^{4} / \pi$. This quantity is constant through the cooling layer, as it is unaffected by changes in the direction of the radiation from the cooling layer. Using Eq. (4), one can integrate Eq. (5) for the profile of the density, from which other quantities can be calculated.

Figure 3(b) shows results of this calculation for the thin-thin case. Here $n=4 / 3$ and $m=2$, which is the correct density dependence and a plausible temperature dependence for experiments with gas, though too weak for astrophysical cases. There is no minimum downstream temperature here, and so the temperature would decrease to zero and the density to infinity. The density ratio shown is the post-shock density increase. The total density increase is larger by the factor produced at the viscous jump, which is 7 here as $\gamma=4 / 3$ was used. (This value of $\gamma$, or something near it, is not only the radiation-dominated value but is also a good estimate for an ionizing plasma.)

In a system for which enough material accumulates downstream to produce a steady final state, the properties of this state are determined from the overall energy flux balance, as is discussed above. The final state becomes only a function of $R_{r}$ (and $\gamma$ ), and has the dependence shown in Figure 4. As in the optically thin case of Figure 3(b), the scale of the density increase with optical depth depends inversely on $R_{r}$. The shape of the cooling layer, in this simple model, depends on the power of density assumed in the opacity. If the opacity is taken to be independent of density, as can occur in some dense materials, then the shape of the curve becomes convex [as opposed to the concave behavior seen in Figure 3(b)]. In simulations 


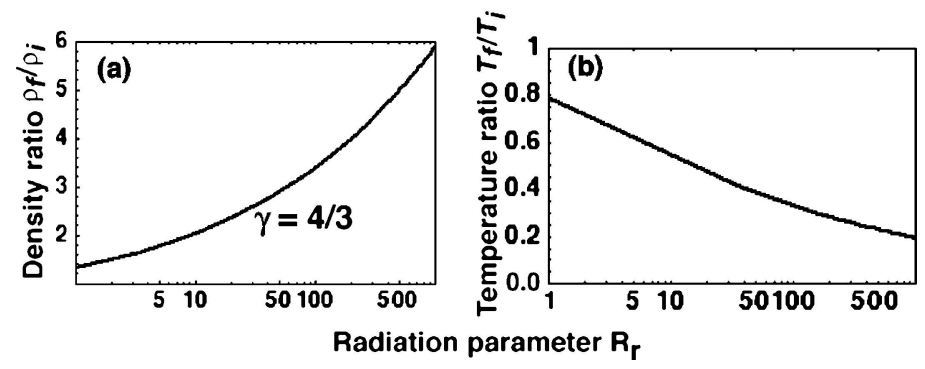

Figure 4. Post shock compression and cooling for an optically thick downstream layer. Results are shown for $\gamma=4 / 3$.

of experiments, we see various shapes, sometimes evolving in time. We do not yet understand all the controlling factors for this.

\section{Conclusion}

We have shown that the detailed properties of radiative shocks depend upon the regime within which they develop, and that this regime can be conveniently characterized in terms of the optical depth of the upstream and downstream regions. At present one might say that shocks with thick upstream and thick downstream regions have been the most explored theoretically, that shocks with thin upstream and thick downstream regions have been the most observed experimentally, and that shocks with thin upstream and thin downstream regions have been the most observed in astrophysics. Yet one can do theory for all the regions and astrophysical examples exist for all regions. So the challenge for various experiments is to access these different regimes and to gain a clear understanding, and clear evidence, regarding the regime studied in any specific experiment.

\section{Acknowledgments}

The author acknowledges useful conversations on this subject with S. Bouquet, D. Ryutov, and M. Hermann. This research was sponsored by the National Nuclear Security Administration under the Stewardship Science Academic Alliances program through DOE Research Grant DE-FG03-99DP00284 and other grants and contracts.

\section{References}

Blondin, J.M., Wright, E.B., Borkowski, K.J. and Reynolds, S.P.: 1998, ApJ 500, 342.

Boireau, L., Clique, C. and Bouquet, S.: 2004, Radiative Shocks in Low-Pressure Gases, Inertial Fusion Science and Applications, Monterey, CA, American Nuclear Society. 
Borkowski, K.J., Blondin, J.M. and McCray, R.: 1997, ApJ 477, 281.

Bouquet, S. et al.: 2004, Phys. Rev. Lett. 92, 22500/1.

Bouquet, S., Teyssier, R. and Chieze, J.P.: 2000, ApJS 127, 245.

Bozier, J.C., Thiell, G., Le-Breton, J.P., Azra, S., Decroisette, M. and Schirmann, D.: 1986, Phys. Rev. Lett. 57, 1304.

Calvet, N. and Gullbring, E.: 1998, ApJ 509, 802.

Chevalier, R.A.: 1997, Science 276, 1374.

Drake, R.P.: 2005, Phys. Plasmas, in preparation.

Ensman, L. and Burrows, A.: 1992, ApJ 393, 742.

Fleury, X. et al.: 2002, Laser Part. Beams 20, 263.

Grun, J., Stamper, J., Manka, C., Resnick, J., Burris, R., Crawford, J. and Ripin, B.H.: 1991, Phys. Rev. Lett. 66, 2738.

Hartigan, P.: 2003, Ap\&SS 287, 111.

Hujeirat, A. and Papaloizou, J.C.B.: 1998, A\&A 340, 593.

Keiter, P.A., Drake, R.P., Perry, T.S., Robey, H.F., Remington, B.A., Iglesias, C.A., Wallace, R.J. and Knauer, J.: 2002, Phys. Rev. Lett. 89, 165003/1.

Koenig, M. et al.: 2001, Radiative shock experiment using high power laser, Shock Compression of Condensed Matter 2001, American Institute of Physics 620, pt2, 1367.

Lamzin, S.A.: 1999, A\&SS 261, 137.

Mihalas, D. and Weibel-Mihalas, B.: 1999, Foundations of Radiation Hydrodynamics, Dover, Mineola.

Reighard, A.B. et al.: 2004, Inertial Fusion and Science Applications, Monterey CA, American Nuclear Society.

Shu, F.H.: 1992, The Physics of Astrophysics: Gas Dynamics, Vol. II, University Science Books, Mill Valley, CA.

Zeldovich, Y.B. and Razier, Y.P.: 1966, Physics of Shock Waves and High-Temperature Hydrodynamic Phenomena, Vol. 1, Academic Press, New York. 